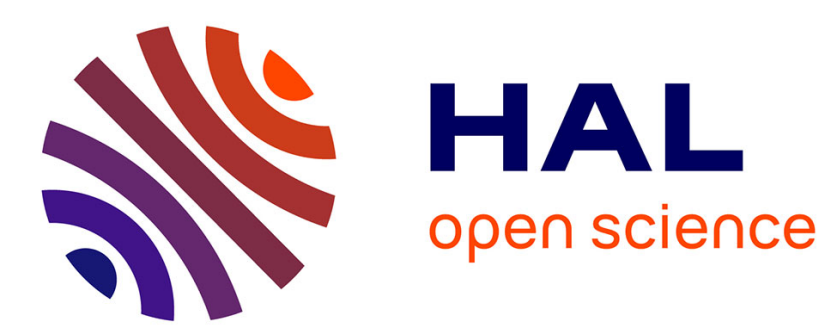

\title{
Artistes et écrivains francophones dans la presse italienne de la Belle Époque: quelques investigations informatiques
}

Julien Schuh

\section{To cite this version:}

Julien Schuh. Artistes et écrivains francophones dans la presse italienne de la Belle Époque: quelques investigations informatiques. Écrivains et artistes de langue française dans les revues italiennes (18801920), Alessandra Marangoni; Julien Schuh, Jun 2016, Padoue, Italie. pp.12-23. hal-01900750

\author{
HAL Id: hal-01900750 \\ https://hal.science/hal-01900750
}

Submitted on 22 Oct 2018

HAL is a multi-disciplinary open access archive for the deposit and dissemination of scientific research documents, whether they are published or not. The documents may come from teaching and research institutions in France or abroad, or from public or private research centers.
L'archive ouverte pluridisciplinaire HAL, est destinée au dépôt et à la diffusion de documents scientifiques de niveau recherche, publiés ou non, émanant des établissements d'enseignement et de recherche français ou étrangers, des laboratoires publics ou privés. 


\section{ARTISTES ET ECRIVAINS FRANCOPHONES DANS LA PRESSE ITALIENNE DE LA BELLE ÉPOQUE : QUELQUES INVESTIGATIONS INFORMATIQUES}

Je souhaitais ouvrir ce dossier par quelques considérations générales sur la manière dont les études littéraires, et en particulier l'analyse des revues, ont changé ces dernières années, suite à la mise en ligne croissante de numérisations et de bases de données, en proposant quelques pistes d'investigation et d'expérimentation à partir des périodiques italiens disponibles en ligne. Je commencerai par revenir sur la nécessité de penser l'histoire culturelle européenne à partir du point d'entrée de la presse, avant de faire le point sur les bases de données de périodiques italiens numérisés disponibles aujourd'hui. Je proposerai enfin quelques exemples d'expérimentations numériques à partir de la problématique de ce dossier, celle de la place des écrivains et artistes francophones dans les revues italiennes du tournant du siècle.

\section{Penser l'histoire culturelle à partir de la presse}

Il y a encore peu de temps, les revues, journaux et autres magazines étaient encore étudiés essentiellement comme des sources pour l'histoire littéraire; on allait repérer les premières publications de tel ou tel article, les critiques de tel ou tel ouvrage, mais la spécificité des revues, leur fonctionnement en tant qu'objets culturels à part entière, n'étaient pas pris en compte. Les choses ont changé, en particulier ces dix dernières années, et la presse est devenu un objet d'étude à part entière, avec parfois même un retournement complet de perspective. Les travaux de Marie-Ėve Thérenty ou d'Alain Vaillant sur une «Civilisation du Journal» ont mené à considérer la littérature moins comme un champ autonome que comme l'un des nombreux phénomènes produit par la culture médiatique, qui gagne au cours du XIX ${ }^{\mathrm{e}}$ siècle une importance considérable en Occident ${ }^{1}$. Par culture médiatique, il faut entendre ce phénomène par lequel tout ce qui est produit d'un point de vue culturel, tous les discours (politiques, sociaux, esthétiques, philosophiques, littéraires) font désormais l'objet d'une forme de dialogue collectif par le biais de la presse. Les grands romans, les grands recueils poétiques dont l'histoire littéraire conserve la mémoire ont d'abord été publiés sous forme de feuilletons, d'extraits, de fragments dans les journaux et les revues. Aux salons de l'Ancien Régime se sont substituées d'autres formes de création collective, par le biais des rédactions, des réunions dans les cafés et cabarets qui donnaient lieu à des créations de périodiques.

Mais l'un des phénomènes les plus importants réside dans la diffusion de ces périodiques à une échelle internationale. Les Belles Lettres n'avaient pas attendu le journal pour proposer des échanges au-delà des frontières; les Lumières furent un phénomène européen qui profita de la solidité des réseaux de diffusion de la correspondance particulière pour fonder un dialogue international ${ }^{2}$. Mais ces dialogues entre particuliers sont rapidement doublés d'une forme de dialogue collectif: utilisant les réseaux postaux, et selon le modèle de la correspondance diplomatique, les premières gazettes qui apparaissent en Europe au cours du XVIII ${ }^{\mathrm{e}}$ siècle permettent à des groupes ou des communautés de particuliers de partager de manière synchronisée des informations. Rapidement, les revues scientifiques, littéraires, politiques se

\footnotetext{
1 Voir Dominique Kalifa, Philippe Régnier, Marie-Ève Thérenty et Alain Vaillant (dir.), La civilisation du journal : Histoire culturelle et littéraire de la presse française au XIXe siècle, Paris, Nouveau monde éditions, coll. Opus magnum, 2011.

2 Voir le projet « Mapping the Republic of Letters », URL $: \underline{\text { http://republicofletters.stanford.edu/ }}$
} 
multiplient ${ }^{3}$. D'une certaine manière, ces revues sont des formes de courriers à destinataires multiples : elles permettent des effets de dialogues à l'échelle de tout un public. Ce phénomène est à mettre en relation avec l'essor des démocraties: de plus en plus de personnes sont concernées par la chose publique, et les discours doivent prendre une tournure globalisante pour toucher le plus de monde. Ces périodiques, permettant des relations au sein de communautés de personnes qui ne se connaissent souvent pas et restent anonymes, sinon pour les comités de rédaction qui disposent des listes d'abonnés, forment ce que Jerrold Seigel appelle des « réseaux de moyens ", c'est-à-dire des réseaux de particuliers qui mettent en commun leurs ressources, à une échelle qui n'est plus celle de la famille ou des réseaux de solidarité traditionnels (l’Église, l'entreprise, le village, le quartier...) mais d'une communauté privée d'intérêts, dont les associations forment un exemple paradigmatique ${ }^{4}$. La manière dont Remy de Gourmont découvre le Symbolisme à travers une revue, La Vogue, est symptomatique de ce phénomène : le périodique permet de toucher des personnes anonymes, par-delà les limites spatiales, afin de fonder des communautés autour de valeurs communes :

J'étais resté assez étranger au mouvement dessiné par mes contemporains, vivant très solitaire en de peu littéraires quartiers, ne connaissant que des noms qu'un écho parfois me renvoyait, ne lisais que des œuvres anciennes, lorsque, tel après-midi sous les galeries de l'Odéon, je me mis à feuilleter la Vogue, dont le premier numéro venait de paraitre. À mesure, je sentais le petit frisson esthétique et cette impression exquise de nouveau, qui a tant de charmes pour la jeunesse. [...] Ce que j’avais écrit jusqu'alors m'inspira soudain un profond dégoût. Je pensai aussi avec amertume au petit journal où, baudelairien innocent, j'avais envoyé des vers, du fond d'un collège de province; et je me disais que, si j’avais persévéré, j'aurais pu écrire dans une de ces émouvantes petites revues et participer directement aux joies que je venais d'entrevoir. J'y parvins, mon orientation littéraire se trouva, en moins d'une heure radicalement modifiée et quatre mois plus tard je publiais Théodat dans la Revue indépendante 5 .

Seigel voit dans ces réseaux la source même de ce que l'on nomme la modernité. Effectivement, l'une des grandes nouveautés introduites par la forme-périodique est celle, justement, de la périodicité. En étant publiés toutes les semaines ou tous les mois, les périodiques créent un horizon d'attente temporel commun pour leurs lecteurs. Il s'agit en définitive d'une réponse aux conditions nouvelles de la vie publique : la modernité implique un renouvellement permanent des valeurs, une accélération des modes et du besoin d'information. Cette temporalité implique aussi un autre rapport au discours: le livre avait provoqué une forme de discours «détemporalisé » ou impliquant une réinterprétation constante (c'était le rôle des institutions comme le clergé ou l'école de choisir un canon et de contrôler son interprétation); les périodiques réinstallent l'imprimé dans l'oralité, qui est moins une manière de produire le discours (oral contre écrit) qu'une temporalité et une forme de communication particulière (on redéfinit qui a le droit d'écrire, de répondre). Cette nouvelle temporalité de la production et de la réception de la culture est précisément ce qui a permis l'essor, lorsque les conditions techniques et politiques ont été réunies, d'une presse en marge des grandes institutions, constituée d'un ensemble de «petits journaux », de « petites revues » financés grâce à une économie autonome de

\footnotetext{
3 Jean Sgard, «La multiplication des périodiques », dans Roger Chartier et Henri-Jean Martin (dir.), Histoire de l'édition française, t. II, Paris, Fayard, 1990, p. 247-255.

${ }^{4}$ Jerrold Seigel, Modernity and Bourgeois Life. Society, Politics, and Culture in England, France and Germany Since 1750, Cambridge, Cambridge University Press, 2012.

${ }_{5}^{5}$ Remy de Gourmont, Promenades littéraires, quatrième série, Souvenirs du Symbolisme et autres études (1912), dixième édition, Mercure de France, 1927, p. 34.
} 
l'avant-garde, petites revues qui irriguent les réflexions des nouvelles générations artistiques à un niveau international, comme le rappelle Stefan Zweig dans ses souvenirs, mentionnant que dans un café de Vienne, à la fin du XIX ${ }^{\mathrm{e}}$ siècle, on pouvait lire le Mercure de France, la Neue Rundschau ou The Studio ${ }^{6}$.

Ce sont précisément ces échanges que je voudrais analyser, en m’intéressant à la manière dont les revues italiennes de la Belle Époque ont «médiatisé » la littérature française — que ce soit par la circulation des textes, en traduction ou dans la langue d'origine, par les comptes rendus et synthèses sur la littérature française, par les échanges de lettres reproduites dans les pages des revues ou par les portraits des littérateurs français.

\section{État des lieux des numérisations de revues italiennes de la Belle Époque}

Pour introduire ces questions, je souhaitais revenir sur les conditions d'analyse et d'étude de la presse aujourd'hui. Il y a quelques années, étudier les revues impliquait d'aller consulter des archives souvent mal présentées, des collections incomplètes, des exemplaires tombant en miette, quand on avait la chance de pouvoir lire des documents papier, la plupart des périodiques étant reproduits sous la forme de microfilms peu lisibles. La masse même de documents imposait des contraintes sur le type d'investigation possible : à moins d'y consacrer des mois, voire des années, il était inconcevable de proposer des études synthétiques couvrant plusieurs titres et de nombreux auteurs; on était réduit aux plongées contrôlées, dans un espace réduit, selon la méthode de l'échantillonnage. Le travail gigantesque de Marc Angenot, dépouillant la totalité de la production imprimée de 1889, représentait la limite haute de ce qui était envisageable — et lui-même s'était autorisé des analyses par échantillon dans certains cas ${ }^{7}$.

Depuis une dizaine d'années, les programmes de numérisation aux niveaux nationaux, souvent financés par des programmes européens comme Europeana, ont permis la mise à disposition au public de centaines de titres de périodiques qui peuvent faire l'objet d'analyses automatisées. Ces outils permettent des recherches ponctuelles, en utilisant les formulaires de recherche en ligne comme celui de RetroNews, mis en place par la BnF, qui permet de repérer des «entités nommées ", c'est-à-dire des noms de personnes, de lieu, d'institutions qui sont repérés comme tels $^{8}$, ou ceux d'Europeana Newspapers'. Mais si l'on souhaite faire des recherches plus systématiques, il faut passer par d'autres moyens ; c'est ce que permet par exemple Europeana en permettant de télécharger la totalité des textes de certains grands journaux européens, afin de faire des recherches avec des logiciels spécialisés ${ }^{10}$. Toutes les institutions ne sont cependant pas prêtes à partager ainsi ces données, alors même qu'elles ont souvent été produites par des programmes de recherche financés par des institutions publiques. Le cas des revues italiennes est ainsi intéressant. Dans l'ensemble, les fonds numérisés disponibles en ligne sont beaucoup moins importants que pour la France ou l'Allemagne. Lorsque ces fonds existent, ils sont souvent assez

\footnotetext{
${ }^{6}$ Cité par Christophe Charle, Discordance des temps: Une brève bistoire de la modernité, Armand Colin, coll. Le temps des idées, 2011, p. 275-276. Sur le phénomène de ces périodiques d'avant-garde, voir Évanghélia Stead \& Hélène Védrine (dir.), L’Europe des revues (1880-1920), estampes, photographies, illustrations, Paris, Presses de l'Université Paris-Sorbonne, 2008 ; Peter Brooker et Andrew Thacker (dir.), The Oxford Critical and Cultural History of Modernist Magazines, vol. I-III, Oxford, Oxford University Press, 2009-2013.

7 Marc Angenot, 1889, un état du discours social, Montréal / Longueuil, Éditions du Préambule, 1989. URL: http://www.medias19.org/index.php?id=11003

${ }^{8}$ URL : http://www.retronews.fr/

9 URL: http://www.theeuropeanlibrary.org/tel4/newspapers

${ }^{10}$ URL : http://data.theeuropeanlibrary.org/download/newspapers-by-country/
} 
mal répertoriés et peu valorisés, et les conditions d'accès aux revues ne permettent pas des recherches précises.

La base $\mathrm{APICE}^{11}$, qui propose de nombreuses numérisations de revues des $\mathrm{XIX}^{\mathrm{e}}$ et $\mathrm{XX}^{\mathrm{e}}$ siècles (Artista, Futurismo, Lacerba, Il Marzocco...) fournit un premier exemple des difficultés que peut rencontrer le chercheur dans l'utilisation des bases de revues. Les liens menant aux titres numérisés ne fonctionnent pas, et il faut passer par des moteurs de recherche externes pour parvenir aux revues numérisées ${ }^{12}$. Le format choisi, djvu, permet de réduire considérablement la taille des fichiers, mais rend problématique toute utilisation de ces numérisations. Il n’y a pas de reconnaissance de texte permettant une recherche dans les textes, et le format djvu rend très difficile l'exportation des images dans un autre format permettant cette reconnaissance de caractères. Enfin, l'interface ne permet de télécharger les fichiers qu'un à un, ce qui rend toute étude particulièrement fastidieuse. La plupart des collections de revues proposées sont par ailleurs incomplètes. Une autre base, les Periodici Italiani Digitalizzati ${ }^{13}$, propose une collection impressionnante. Ici encore, les choix techniques rendent malheureusement cette base quasiment inutilisable : même si ces périodiques sont depuis longtemps dans le domaine public, la base les présente comme des objets sur lesquels l'institution détiendrait un droit à l'image. Les pages présentent toutes un filigrane BIASA, et tout téléchargement est impossible. La recherche plein texte n'est pas disponible. On est ainsi face à une mine d'information qu'il faut dépouiller page à page, comme devant un microfilm, alors que l'interface, particulièrement rigide et lente, rend toute manipulation difficile. Le même problème rend l'utilisation de la base de données CIRCE ${ }^{14}$ difficile : les numérisations de nombreuses revues culturelles du XX $\mathrm{XX}^{\mathrm{e}}$ siècle $(\mathrm{La}$ Balza futurista, Il Convito...) ne sont accessibles que page à page. Certains projets s'attachent à une revue en particulier. La revue Emporium est ainsi disponible par le biais du Progetto Emporium ${ }^{15}$, qui propose des classifications thématiques des textes et des illustrations, mais pas de téléchargement direct des numéros, qui sont cependant consultables en ligne. La Voce et Il Marzocco peuvent être consultés sur le site du Gabinetto G.P. Vieusseux ${ }^{16}$.

Les revues italiennes sont également disponibles dans des bases de données internationales. La bibliothèque numérique HathiTrust ${ }^{17}$, fondée par une association d'institutions américaines qui ont fait numériser leurs fonds par Google Books et ont mis en place une plateforme numérique de valorisation de ces imprimés, met ainsi à disposition des internautes plusieurs revues italiennes numérisées. HathiTrust est malheureusement limité par des accords avec Google, qui l'empêche de diffuser ses fichiers hors des États-Unis; il faut donc souvent utiliser des moyens détournés pour avoir accès aux numérisations ${ }^{18}$. Paradoxalement, c'est la base qui offre le plus de numérisations de revues italiennes, comme Flegrea, La Rassegna Internazionale della letteratura e dell'arte contempranea, Emporium, Leonardo... avec de rares lacunes. Enfin, quelques revues italiennes sont disponibles sur Gallica (Anthologie-Revne de France et d'Italie) et sur la base du Blue Mountain Project $^{19}$, qui donne un très bon accès aux revues d'avant-garde entre 1890 et 1930, mais qui n'a numérisé qu'une partie des numéros de Poesia.

\footnotetext{
${ }^{11}$ URL : http://apicesv3.noto.unimi.it/site/

${ }^{12}$ En tapant par exemple « site:http://apicesv3.noto.unimi.it/ futurismo ».

13 URL : http:// periodici.librari.beniculturali.it/

${ }^{14}$ URL : http://circe.lett.unitn.it/main page.html

15 URL: $\underline{\text { http://www.artivisive.sns.it/progetto emporium.html }}$

16 URL: $\underline{\text { http://www.vieusseux.it/coppermine/index.php }}$

17 URL: https:// catalog.hathitrust.org

${ }^{18}$ Voir quelques exemples de manipulation sur le site PRELIA, URL : https://prelia.hypotheses.org/457

${ }^{19}$ URL : http://bluemountain.princeton.edu/index.html
} 


\section{Expérimentations}

À partir du texte de ces revues numérisées, j'ai constitué un corpus intégrant tous les textes disponibles des numéros de sept revues italiennes de la Belle Époque : Emporium (uniquement les années 1895-1896), Anthologie-Revue de France et d'Italie (1897), Il Marzocco (uniquement entre 1898 et 1901), Flegrea (1899-1901), La Rassegna Internazionale della letteratura e dell'arte contempranea (19001901), Leonardo (1903-1907), Poesia (1905-1909). Il est possible d'extraire de ce corpus, de manière automatisée, un certain nombre d'informations statistiques, relevant principalement de l'analyse de réseaux et de la sociologie de la littérature.

On peut commencer par repérer la manière dont les revues se positionnent les unes par rapport aux autres, par le biais de l'analyse factorielle des correspondances ${ }^{20}$ (fig. 1). Ce calcul permet de classer les revues sur un plan bidimensionnel en fonction de leur vocabulaire : les titres dont les lexiques sont proches sont regroupés dans l'espace du graphique. On constate la position marginale de l'Anthologie-Revue, qui publie la moitié de ses textes en français; ceci explique que la revue la plus proche d'elle soit Poesia, qui publie également beaucoup de textes directement en français. Poesia et Anthologie-Revue forment ainsi un groupe linguistique, qui se distingue d'une part du groupe constitué par Emporium et Leonardo, d'autre part du groupe de Flegrea, Il Marzocco et La Rassegna. On pourra vérifier si ce classement concorde avec la manière dont les écrivains et artistes francophones sont représentés dans ces revues.

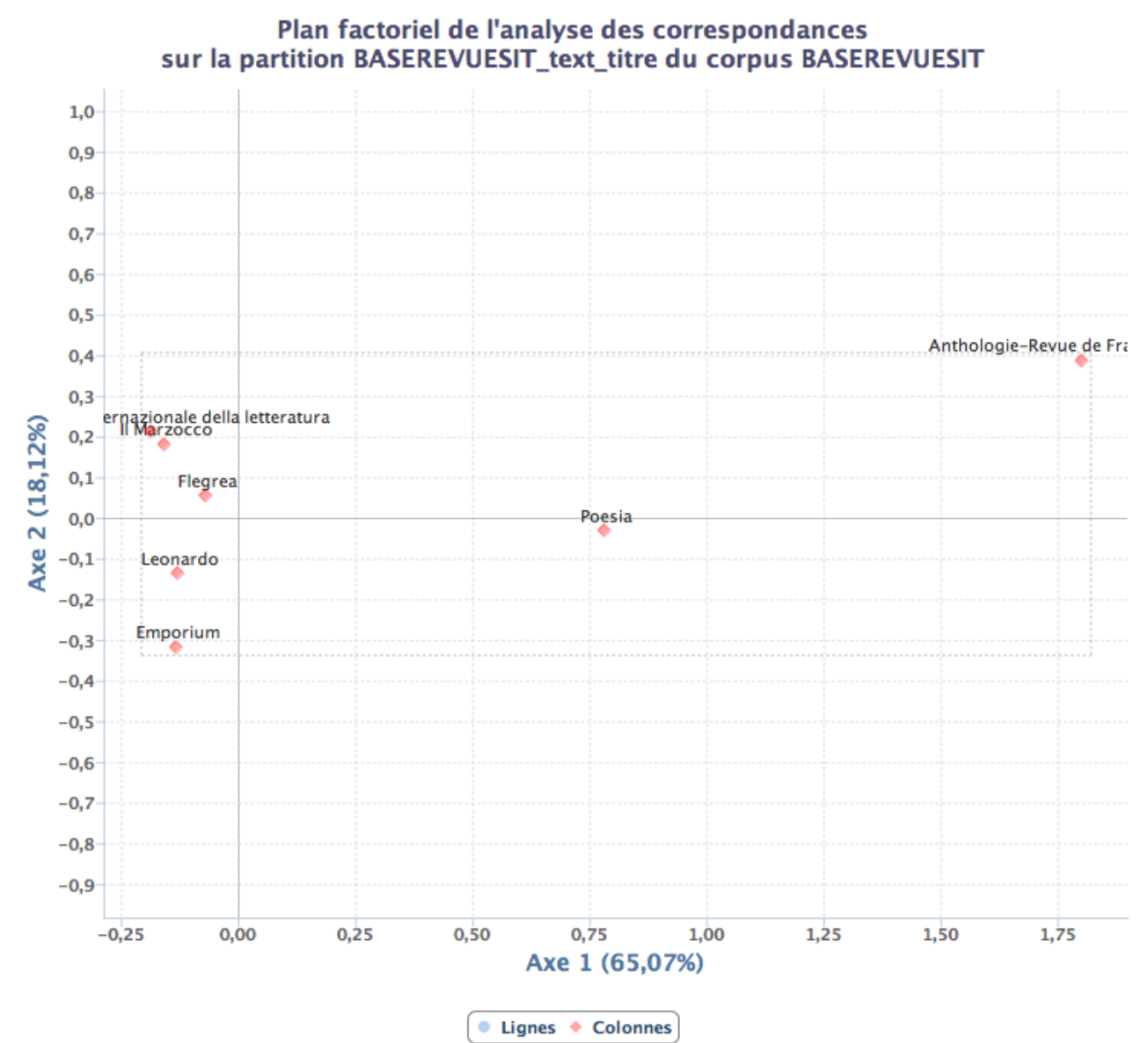

${ }^{20}$ J'ai utilisé l'outil d'analyse lexicométrique TXM, URL : http://textometrie.ens-lyon.fr/ 
Fig. 1.

Pour repérer les auteurs francophones dans les revues italiennes de cette époque, je suis parti de la liste des personnalités littéraires citées dans l'Enquête sur l'évolution littéraire de Jules Huret de 1891 (d'abord publiée dans le journal L'Écho de Paris), qui livre un index précisant le nombre de mentions de chaque auteur dans le volume ${ }^{21}$. J'ai considéré que cet index constituait une sorte de cliché du poids médiatique de chacun de ces auteurs dans le champ littéraire français de l'époque, que j'ai utilisée pour interroger le texte des revues italiennes et y dénombrer les mentions d'auteurs français, en tenant compte de l'italianisation de certains noms (Paul Adam devient ainsi parfois Paolo Adam). D’un point de vue méthodologique, je n'ai pas pu différencier strictement les mentions d'auteurs de leur signature (même si en réalité les signatures, souvent en majuscules, n’ont pas été prises en compte). Pour équilibrer les deux corpus, j'ai multiplié par trois le nombre de mentions dans l'enquête de Huret, dont le texte est bien plus court que celui des revues italiennes.

En ne considérant que les auteurs français mentionnés plus vingt-cinq fois, on obtient une liste de 46 noms, de Balzac à Zola (fig. 2), dont on peut comparer l'importance dans l'enquête de Huret (en gris foncé dans le graphique) et dans les revues italiennes (en gris clair). Dans l'ensemble, les poids médiatiques des écrivains sont étonnamment semblables en France et en Italie. Parmi les personnalités les plus mentionnées, on trouve par exemple Mallarmé, qui bénéficie dans les deux pays d'une forme de surreprésentation médiatique par rapport aux lecteurs de ses œuvres. Cela tendrait à confirmer l'idée d'une forme particulière de renommée littéraire à cette époque, à un niveau international, liée avant tout au caractère aisément reconnaissable d'une personnalité littéraire qui rassemble dans son nom un ensemble de valeurs (souvent clivantes, comme c'est le cas aussi pour Zola). Avec des outils d'analyse des cooccurrences, on peut examiner les termes qui apparaissent le plus fréquemment à proximité du nom de Mallarmé dans les revues italiennes: on remarque qu'il est souvent cité dans une constellation avec Verlaine, Villiers, Rimbaud et les courants du Parnasse et du Symbolisme. Il est représentatif, et on le nomme sans même l'avoir beaucoup lu. Il est intéressant de constater qu'une forte présence médiatique en France est souvent corrélée à une forte présence à l'étranger: la célébrité s'exporte, traverse les frontières, confirmant la porosité des espaces médiatiques nationaux fins de siècle.

${ }^{21}$ Jules Huret, Enquête sur l'evolution littéraire, Paris, Bibliothèque Charpentier, 1891. 


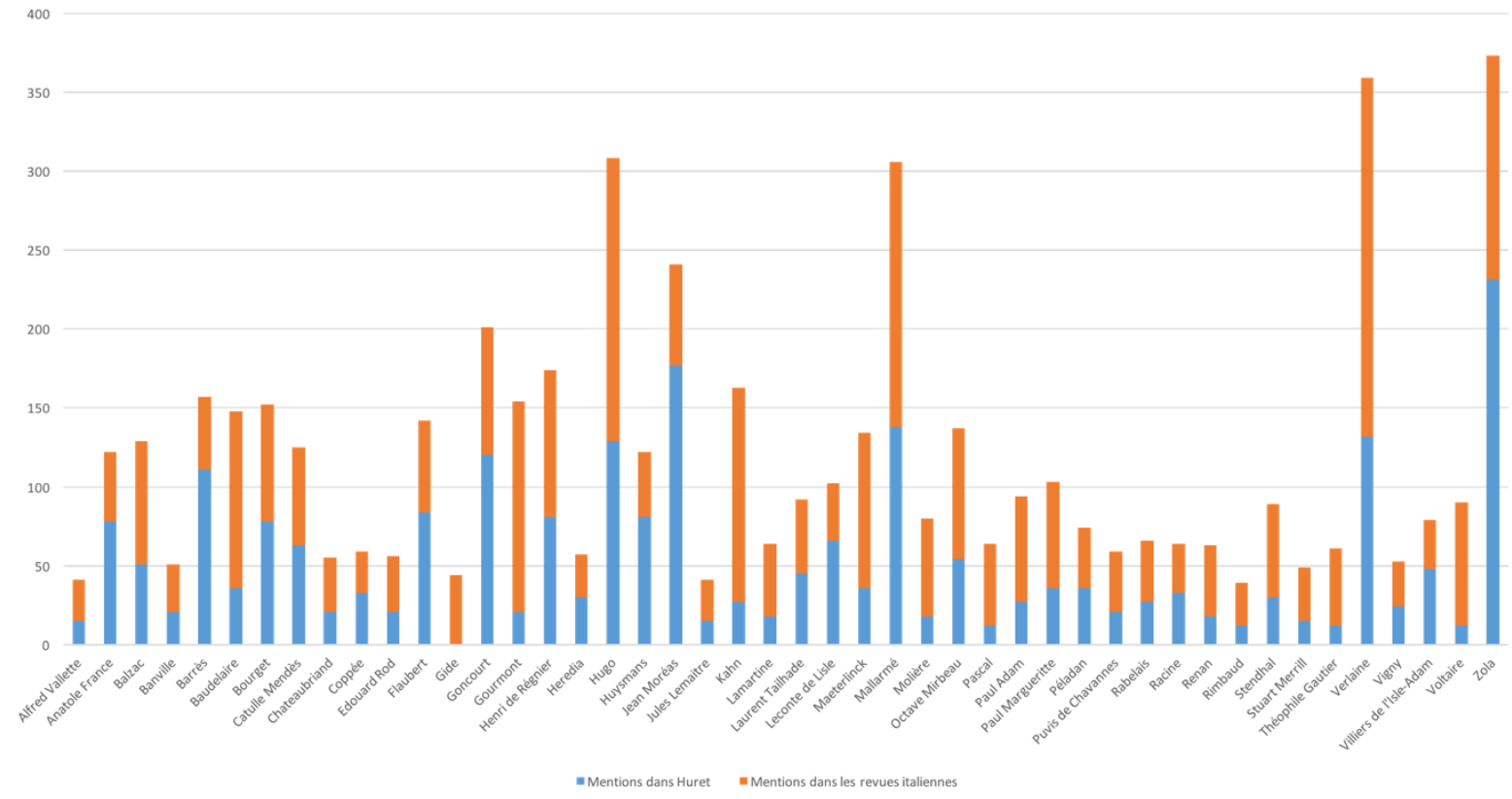

Fig. 2.

On remarque tout de même des spécificités nationales. Remy de Gourmont est par exemple très présent dans les revues italiennes par rapport à son poids dans les réponses à Huret; Alexia Kalantzis a bien montré son rôle dans l'internationalisation de la littérature symboliste, qui peut expliquer ce décalage ${ }^{22}$. On peut peut-être le rapprocher d'un autre écrivain cosmopolite à sa façon, Voltaire, bien plus cité en Italie qu'en France. À l'inverse, une personnalité comme Jean Moréas ne bénéficie pas en Italie de la même présence dans la presse qu'en France; il faut prendre en compte qu'en 1891, le nom de Moréas avait fait l'objet d'une campagne pour affirmer l'existence du Symbolisme autour de sa personne, son empreinte médiatique était alors particulièrement forte en France. Verlaine est bien plus cité dans les périodiques italiens que dans la presse française : si les Symbolistes ont tendance à lui préférer Mallarmé ou Villiers, il écrase ses rivaux à l'étranger. Toutes les mentions ne se valent cependant pas. L'importance d'un nom comme celui d'Alfred Vallette dans les revues italiennes est ainsi à nuancer: elle est essentiellement liée aux réclames. Toutes les occurrences du nom du directeur du Mercure de France dans les revues italiennes apparaissent dans des pages mentionnant sa revue. Ce graphique ne rend pas compte des écrivains qui n'étaient pas encore connus en France en 1891, tels Jarry, Valéry, Claudel, Gide, Apollinaire, Proust... L'importance médiatique de Gide en Italie est cependant impressionnante: tout jeune encore, dans la génération qui débute au début des années 1890, il est déjà bien représenté dans les revues italiennes. Valéry de son côté n’a droit qu'à une mention dans Poesia dans les années 1920.

On peut s'intéresser aux représentations spécifiques à chaque revue, qui ne donnent pas le même poids à tous les écrivains, en fonction de leurs choix esthétiques ${ }^{23}$. Hugo, Mallarmé, Verlaine, Zola sont mentionnés de manière presque équivalente dans l'ensemble du corpus ; par

22 Alexia Kalantzis, Remy de Gourmont créateur de formes. Dépassement du genre littéraire et modernisme à l'aube du XXe siècle, Paris, Champion, 2012.

${ }^{23}$ Les graphiques en couleur qui accompagnent cet article sont disponibles en ligne sur le site www.prelia.fr 
contre, un écrivain comme Gustave Kahn, inventeur du vers libre, n'apparaît quasiment que dans Poesia, comme le poète Henri de Régnier, alors que Gourmont est absent de la revue de Marinetti, comme d'Emporium. La Rassegna internazionale mentionne principalement le canon littéraire des générations précédentes (Balzac, Flaubert, Hugo); c'est aussi la revue qui valorise le plus les romanciers à succès et les « psychologues » (Bourget, Rod, Mirbeau, Zola). Gourmont y est assez isolé. Leonardo fait des choix très particuliers (Pascal, Voltaire, Maeterlinck, Renan, Barrès, Gourmont) qui la distinguent des autres revues et confirme son caractère idéaliste. Verlaine est relativement peu présent dans Poesia, tout comme Mallarmé, ce qui corrobore leur côté « daté» pour cette revue.

Quels facteurs permettent le mieux de rendre compte de la disparité des mentions d'écrivains français dans les revues italiennes? Une visualisation sous forme de constellation permet de faire apparaitre certaines particularités ${ }^{24}$ (fig. 3). Ici, les différences de représentation des auteurs francophones dans les revues italiennes prennent la forme d'un réseau; la taille des liens est proportionnelle au nombre de citations. Les noms liés aux mêmes revues sont rapprochés dans l'espace du graphique, ce qui permet de le lire comme une image du champ littéraire français dans l'espace des revues italiennes. Verlaine et Mallarmé apparaissent dans un groupe formé par les grands noms du XIX ${ }^{e}$ siècle (Baudelaire, Hugo, Lamartine, Gautier); l'autre groupe de "Classiques », Pascal, Molière, Voltaire, sont du côté de Leonardo, d'Il Marzocco et de La Rassegna internazionale, avec Flaubert, Anatole France et Lemaitre. Autour de Poesia se regroupent les Symbolistes et les Parnassiens. Une continuité esthétique et idéologique se dessine; ces regroupements montrent comment l'histoire littéraire française est reconstruite dans les revues italiennes.

${ }^{24}$ Visualisation réalisée avec le logiciel Gephi, URL : https://gephi.org/. 


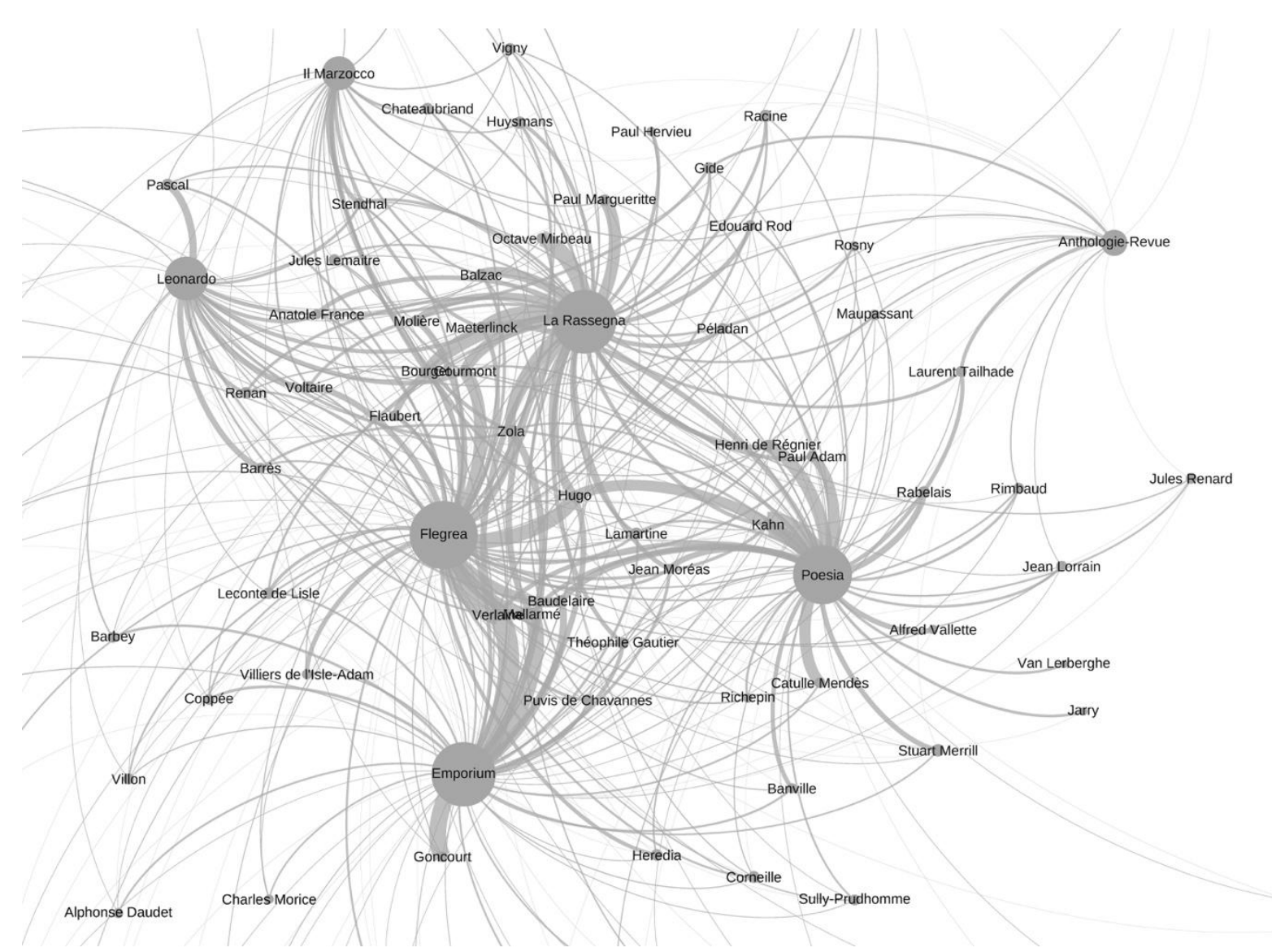

Fig. 3.

On remarque que les oppositions en groupes de revues que nous avions vues à partir de l'analyse linguistique de leur texte sont en partie confirmées: Poesia et Anthologie-Revne sont classées ensemble, mais ici les deux autres groupes sont constitués par Emporium et Flegrea d'un côté, Il Marzocco et La Rassegna de l'autre, Leonardo constituant un groupe à part. Le rapprochement entre Emporium et Flegrea est lié à un groupe d'auteurs (Mallarmé, Verlaine, Baudelaire, Hugo...) formant une sorte de canon poétique ; celui de La Rassegna et d'Il Maržocco se fonde sur des romanciers (Stendhal, Mirbeau, Balzac...). La mise en évidence des genres principaux dans lesquels écrivent ces auteurs permet de confirmer l'importance de ce facteur générique dans la structuration de l'espace des revues italiennes: les dramaturges et les romanciers forment un bloc assez cohérent dans la partie supérieure du graphique, les poètes dans la partie inférieure. Il y aurait donc une spécialisation générique des revues, expliquant la présence ou l'absence de certains auteurs dans leurs pages.

La reconstruction du champ littéraire français dans les revues italiennes permet ainsi de confirmer l'existence d'une forme de champ médiatique international, structuré principalement par des oppositions génériques et générationnelles. Cette structuration recoupe en grande partie l'analyse du champ littéraire proposée par Bourdieu entre la " grande production » orientée vers le grand public (le roman) et la «production pure » destinée au marché restreint des littérateurs (la poésie), elle-même divisée «entre l'avant-garde et l'avant-garde consacrée $e^{25}$ »- ce qui laisse

${ }^{25}$ Voir Pierre Bourdieu, Les règles de l'art: Genèse et construction du champ littéraire (1992), Éditions du Seuil, coll. Points Essais, 1998, p. 204-205. 
ouverte la question de savoir si ces revues héritent d'une structuration préexistante ou si elles contribuent à son élaboration.

D’autres expériences seraient possibles (repérage de thématiques, évolutions chronologiques, repérage des reprises de textes entre revues, voire des traductions par le biais de traductions automatisées). J'espère que ces quelques expérimentations permettront de mieux comprendre les analyses de cas particuliers évoqués dans ce dossier.

Julien SCHUH

Université Paris Nanterre 